\title{
Practising what we preach
}

\author{
Kenneth C Calman
}

\section{Correspondence to}

Professor Kenneth C Calman, Department of Medicine,

University of Glasgow, Glasgow G12 8QQ, UK;

Kenneth.Calman@glasgow.ac.uk

Received 4 September 2014 Revised 25 September 2014 Accepted 29 September 2014

\section{ABSTRACT \\ The question of what makes 'good medical ethics' is explored focusing on the principles and values of the clinical professions, how these are integrated into patient care and shared with the wider public and on the central importance of good communication. The need for an effective learning base at all stages in professional careers is emphasised and the importance of measuring and monitoring outcomes of care and public health issues from an ethical perspective is discussed while acknowledging the difficulties of doing so. The possibility of establishing a wider forum to discuss this topic is mooted. The essay emphasises the importance of practising what we preach.}

\section{INTRODUCTION}

This essay is the result of a challenge presented by the 'set question' for this 40th anniversary issue of the Journal of Medical Ethics, 'What is it to do good medical ethics?' The question raises many issues, each of which deserves more extensive comment than permitted in a short essay. However, the first issue that must be addressed is some sort of a definition of 'good medical ethics'-and I offer an initial draft account. I follow this with a series of thoughts on related issues, including the important question of how medical students and doctors learn about medical ethics and the relative roles of health professionals and of specialist teachers, including those from various humanities backgrounds. I reflect on a variety of related issues, including the role of specialists in medical ethics, the importance of good communication to good medical ethics, the ethical relevance of the uncertainty, risk and need for good evidence characteristic of medical practice and the sometimes uncertain ethical relationship of clinical practice and medical research. I emphasise the importance of the clinical reflection and 'practical wisdom' of health professionals concerning the ethical issues that they face every day and that differentially arise in their various specialties and I try to bring these issues together within the terms of my initial definition; and I ask whether or not we actually practise what we preach.

\section{SOME BACKGROUND ISSUES}

My background is relevant as to how I answer the question. I began my clinical career almost 50 years ago, first as a trainee vascular and transplant surgeon, morphed into an oncologist with an interest in palliative care and then became involved in public health. Each task was associated with a different group of people and a wide range of ethical issues.
One of the striking features over the years is how medicine has evolved and a whole new range of new treatments, diagnostic tests and diseases has emerged. Clinical care has become more complex and more effective, with each of these new issues having their own ethical issues. At the same time, new methods of teaching and discussion have been used and tested and new journals have been launched. In addition, the ethical dimension has become more closely linked to the broader field of the medical humanities. Thus, over my own clinical lifetime much has changed and improved in both patient care and public health.

In particular, there has been a significant, and welcome, rise in patient and public involvement. The power of the patient is now clear and there is much greater discussion and debate with patient, family and the wider community.

\section{WHAT IS GOOD MEDICAL ETHICS? A POSSIBLE DEFINITION}

The following proposal provides the starting point for the discussion before we consider whether or not we practise what we preach.

Good medical ethics is practised when there is a clear agreement on the principles and values of the medical profession and that these are seen to be an integral part of patient care. These are shared with patients and the public and communication is central both to the patient consultation and the public understanding. An effective learning base in ethics for all in the medical and related professions is in place at all stages in their careers. There is a method of monitoring the quality of practice and of learning from this experience and this is an established part of good medical practice.

To expand on this definition, the next few sections look at statements within this proposal before coming to some final conclusions.

\section{WHAT IS MEDICAL ETHICS?}

Medical ethics is a subset of general ethical issues particularly concerned with health and medicine. The subject thus draws its intellectual power and experience from centuries of considering the values of life and living from many different facets and is linked to wider issues such as the condition of human society, political philosophy, human rights, freedom, quality of life and how we live. At the heart of medical ethics is the value base from which decisions are made. Ethical decision making is not arbitrary, but different groups of people will come from different value bases and thus may have different values and beliefs. As an example, abortion raises different issues in different religious groups,
To cite: Calman KC. J Med 
as do end-of-life care and assisted dying. These values relate to both individuals and populations.

In medical terms, these values and beliefs are often codified into oaths or statements, most common being the Hippocratic Oath, or one of its modern variants. These are very powerful statements often voiced publicly by each graduate at ceremonies for medical students and other professional groups. Such ethical statements give guidelines only and in making an individual decision they may not give all the answers. However, the general thrust is that doctors, and other health professionals, agree to do the best for patients and not to do harm. They set the tone and send a signal that values are important. These statements are seen to be integral to patient care and the health of the public and not just a useful add-on.

There is a subset of this question; what is the role of the specialist in medical ethics? Quite properly there are those who spend much of their time thinking, teaching and writing about medical ethics. What do they contribute to the overall care of patients and their families? Should they come on every ward round to help decision making? Or be part of the clinical discussions on future care of patients? Or is their role simply to teach the generalities of ethics and leave the clinician to come to a decision and get on with it? In which case how do we know what is 'good' medical ethics? Is it only when things go wrong that we recognise a problem?

In many instances, the institution in which the patient is being cared for will have an ethical committee to oversee decision making and in particular those aspects of care related to research and clinical trials but also increasingly a clinical ethics committee to advise on ethically contentious issues in clinical practice. This gives assurance to patients and their families that the issues have been carefully considered, all of the factors have been taken into account and that patients can have confidence that the clinical procedure or treatment will be of benefit to the individual or to a wider group of patients. There are also national and international ethical groups who publish regular reports on ethical topics. Such groups bring together health professionals and ethical specialists to discuss and debate and provide guidance.

A further aspect of clinical decision making is to consider the role of uncertainty, risk and the evidence base. People are individuals and some will not respond to treatments and the risks of a treatment causing harm may be real. Hence, the need for full discussion with patients and their involvement in decision making. The evidence base is critical, but it can only become a strong evidence base if there has been research, some of it perhaps though clinical trials with a placebo. This sometimes makes it difficult to separate research ethics from clinical practice. A current example of this might be the treatment of Ebola virus with drugs which have not been tested, but may offer hope. This was widely considered to be ethically justifiable.

There are therefore, within each medical specialty, particular issues which require consideration; whether it is in paediatrics, palliative care, complex surgical procedures or the care of the elderly. The clinical teams involved will have thought through these issues and will demonstrate the practical value of such ethical reflection in ward rounds, clinics and the operating theatre. For those in training, these are especially valuable settings.

\section{HOW DO YOU LEARN ABOUT MEDICAL ETHICS?}

Learning is a key part of doing good medical ethics. Over the last 30 years or so, the ways of learning and teaching the subject area have changed considerably. Initially there was not much formal teaching at all, and a great deal was learnt at the bedside or the clinic, listening and watching and taking part in case discussions. This remains, of course, a fundamental part of learning. Lectures were introduced and student societies and groups set up to discuss ethical issues. Now most medical schools will have some form of course on the subject. This is to be encouraged, but there are dangers, in that it may be seen that the professor has all the answers which are then written down by the student as the 'truth'. In my own teaching, for example, I usually begin with a statement such as "don't believe everything I say, it is your personal view which matters". The trainees must think it through for themselves. There is also the danger that medical ethics might also be seen as something separate from normal clinical practice.

It is possible, even with a large class, to encourage debate and personal decision making. By using case studies, press reports, poems and literature as triggers, the class can be asked to go into small groups, even in a lecture theatre setting, to discuss the issue and report back. This method begins to show differences and conflicts in the class, and what indeed might be expected in real clinical work in future.

Postgraduate education has also become much more involved in ethical debate, in local meetings, conferences and seminars. These are to be encouraged and expanded. The learning can be enhanced further if the views of patients and the public are added. Some clinical organisations have a public membership and they are encouraged to be part of that debate during conferences and seminars and this adds enormously to the learning experience.

Over the last 30 years or so, books, journals and publications have also given rise to more food for thought. As has been noted, national and international bodies with an ethics remit have helped to lead the debate and ensure that topics are fully debated and discussed and all points of view explored. A good example of this is the current debate on end-of-life care and assisted dying. There are different points of view and these need to be raised and discussed, including of course that of the patient and the family. Current issues might include the cost of drug therapy, palliative care and the use of complex and expensive life-saving procedures. All of this involves a broader debate with the public on ethical issues.

A further dimension has been the wider involvement of the humanities in medicine and in medical education. This movement began around 30 years ago and is now a valuable input into ethics and clinical care. It tries to see patients and communities holistically (a central part of care) and uses, in addition to the philosophical framework of ethics, the arts and humanities such as literature, the visual arts, dance and movement. This has brought different dimensions to thinking about ethical issues and in uncovering what matters most to patients. There is no dichotomy here, simply an expansion of the knowledge and learning base of medical ethics.

In summary, medical education has changed much over the last three decades, and this has been to the benefit of those who feel that medical ethics is an important part of the work of the doctor and other health professionals. It also gives a responsibility to those who assist learning to evaluate their approaches, develop the evidence base and publish the outcomes in journals and books. Only in this way can we ensure that medical ethics continues to be a key part of professional education, and that the methodology used is effective.

\section{SO WHAT DOES GOOD MEDICAL ETHICS MEAN?}

Here, we return to the statement at the beginning of this essay. The first issue to be raised was the need to have to a clear 
statement of the values of the medical profession. This is available in many different forms and should be agreed by the profession and shared with the public. It should be set out as a series of principles-such as the General Medical Council documents-recognising that in particular circumstances these may not fit well and will require further discussion. As medicine advances with new treatments and diagnostic techniques, it will become ever more complex and new ethical issues present new problems.

This is also why the public need to be kept up to date, not just with medical advances, but with their consequences. They also need to be aware of the ethical values agreed by the profession and these should be displayed in clinical settings, providing ways in which patients and the public can interact. This stresses the importance of communication, both with the patient and family, and with the wider public. Major national and international organisations who have a specific interest in ethics, and who debate such topics regularly, also have a responsibility to make such views widely known and readily available.

The public should be helped to become more aware of the risks associated with treatment and investigations. For example, recent discussions on the cost of cancer drugs and their level of efficacy is an important one. An individual patient with cancer may see this issue differently from the broader public. How can good medical ethics assist in this discussion? Trying to explain the concept of the 'greater good of the greatest number' to a patient who might benefit from a costly drug but cannot get it is not easy. The key, however, is involvement of the patient and the public. The same issues arise in the conduct of clinical trials, where one arm of the trial may be less effective than the other. The patient here is contributing to something more than their own care and to the benefit of others. Similar issues arise in the public health sphere where hazards to the public, for whatever reason, need to be explained and action (or no action) justified.

Thus, a clear professional view of good medical ethics and greater public and patient involvement are prerequisites for good medical ethics. This statement, however, makes an assumption that we know what good medical ethics is and how this might be monitored. This will be discussed in more detail later.

The second issue which was discussed earlier was the learning process in understanding and practising good medical ethics. This remains critical and each group of learners, undergraduate or postgraduate, deserves to have an appropriate opportunity to learn and to question. This dialogue is at the heart of the process of learning. As part of this, patient and public views need to be fed in. Medical ethics, as has been mentioned, is not a separate and isolated option, taught in a separate class, but integral to clinical care. It should be part of routine clinical management that the ethical dimension is covered and active clinical staff should be involved in the presentations to learners.

A further aspect of this is the involvement of patients and communities. This can be done in a variety of ways, including the use of patient views in learning. Public meetings can be another way of exposing the thoughts of communities and the public on a variety of topics. This allows discussion of risk, the evidence base and explaining how decisions are made. This is not an easy process but could be seen as part of good medical ethics. There should also be a recognition that there will be different values in any group of people and that 'the doctor knows best' may be an outdated concept!

Thus, good medical ethics has a number of components:

- A clear statement of values

- Medical ethics seen as an integral part of the process of care

- Involvement of patients and the public

- Appropriate education and learning at all levels

But this is not sufficient. There needs to be a continuing process of monitoring the effectiveness of the actions taken. In broader national terms, there is a requirement for clear, hard outcomes which can be measured and published and comparisons made between clinical teams. Such work is time consuming and potentially expensive. To measure outcomes is more difficult in the area of medical ethics where the outcome may be related to quality of life or satisfaction with the care given. In some instances, where mistakes have occurred, then a poor outcome of care is obvious. In a similar way, patient complaints referred to a variety of public bodies provide another way of ensuring that problems are raised and learning occurs from the process of review.

This, however, leaves the onus on the patient or family to take action and complain and is a passive process for the doctor. The current drive for continuing professional development and re-accreditation provides more individually focused methodology in which the doctor or the team can continually examine their practice and outcomes of care. The argument here is that this should include, in addition to the mortality rates, problems with treatment, days in hospital and many other aspects, the ethical issues faced and whether or not they have been effectively discussed and with appropriate patient involvement. This is the proactive part of considering the basis of good medical ethics. This leads to the final bullet points:

- Effective monitoring of practice and the related learning experiences

- Continual examination of clinical practice related to medical ethics

The practice and knowledge base of medical ethics has changed considerably over the years, in line with changes in medicine and public health, though the basic principles have remained essentially the same. It will continue to change and at the same time become more accountable to patients and the public. All of the elements of good medical ethics are present in current clinical practice. However, we need to ensure that they are all active and effective. The process of integration and review noted earlier will ensure that the healthcare professions will continue to provide good medical ethics to those under their care, and that we practise what we preach.

One final issue which I raise is to propose the establishment of a committee or commission whose deliberations would enlighten the professions, the patients and the politicians. Its purpose would be to answer the question, 'What is good medical ethics?' Such a commission could be established under the auspices of an existing organisation and would include a wide range of people from different backgrounds. This might help to bring a broad perspective on the subject and would also provide an opportunity to work with patient groups and ensure that their views were taken into account.

Competing interests None.

Provenance and peer review Commissioned; internally peer reviewed. 